\title{
TRIPLE ORTHOGONAL SERIES
}

\author{
by ROBERT FEINERMAN
}

(Received 19 July, 1977)

In a number of recent papers, we have developed an abstract approach to dual orthogonal series (see [1], [2], [3], and [4]). Such series arise in crack theory, heat transfer, etc. In this paper, we generalize these results to triple orthogonal series. We also show, via a counterexample, that, surprisingly, the results in the dual case are not generalizable as completely as expected.

Notation. In this paper we shall always have:

(1) $H$ is a real separable Hilbert space.

(2) $\mathbf{P}$ and $\mathbf{Q}$ are orthogonal subspaces of $H$ (i.e., $H=\mathbf{P} \oplus \mathbf{Q}$ ).

(3) $\mathbf{P}_{1}, \mathbf{P}_{2}$, and $\mathbf{P}_{3}$ are mutually orthogonal subspaces of $H$ (i.e. $H=\mathbf{P}_{1} \oplus \mathbf{P}_{2} \oplus \mathbf{P}_{3}$ ).

(4) $P$ and $Q$ are projection operators from $H$ onto $\mathbf{P}$ and $\mathbf{Q}$ respectively, so that $P+Q=I$, the identity operator.

(5) $P_{1}, P_{2}$, and $P_{3}$ are projection operators from $H$ onto $\mathbf{P}_{1}, \mathbf{P}_{2}$, and $\mathbf{P}_{3}$ respectively, so that $P_{1}+P_{2}+P_{3}=I$.

(6) $\left\{\varphi_{n}\right\}_{n=1}^{\infty}$ is a complete orthonormal sequence in $H$.

The main results of [1] and [3] can be written as:

THEOREM A. Let $\left\{a_{n}\right\}_{n=1}^{\infty}$ be a sequence of positive numbers and let $\psi_{n}=P \varphi_{n}+a_{n} Q \varphi_{n}$. Then $\left\{\psi_{n}\right\}_{n=1}^{\infty}$ is complete in $H$.

THEOREM B. Let $\left\{a_{n}\right\}_{n=1}^{\infty}$ be a sequence of positive numbers such that $0<m \leq a_{n} \leq M$. If $\psi_{n}=P \varphi_{n}+a_{n} Q \varphi_{n}$, then $\left\{\psi_{n}\right\}_{n=1}^{\infty}$ is an $\ell^{2}$ basis in $H$, i.e. given $F \in H$ there is a unique sequence $\left\{K_{n}\right\}_{n=1}^{\infty}$ such that $\sum_{n=1}^{\infty} K_{n} \psi_{n}=F$ (in the norm of $H$ ) and, moreover, $\left\{K_{n}\right\}_{n=1}^{\infty}$ is square summable.

It is these two theorems for which we shall try to find analogues in the case when $H$, instead of being split into two subspaces, is split into three subspaces. As we said earlier, a surprising factor arises in the analogues of both theorems.

THEOREM 1. Let $\left\{a_{n}\right\}_{n=1}^{\infty}$ and $\left\{b_{n}\right\}_{n=1}^{\infty}$ be sequences such that

$$
1-\frac{1}{\sqrt{2}} \leq a_{n} \leq 1+\frac{1}{\sqrt{2}} \text { and } 1-\frac{1}{\sqrt{2}} \leq b_{n} \leq 1+\frac{1}{\sqrt{2}} \text {. }
$$

If $\psi_{n}=P_{1} \varphi_{n}+a_{n} P_{2} \varphi_{n}+b_{n} P_{3} \varphi_{n}$, then $\left\{\psi_{n}\right\}_{n=1}^{\infty}$ is complete in $H$.

Proof. Let $a_{n}=1-\epsilon_{n}$ and $b_{n}=1-\delta_{n}$. Then $\left|\epsilon_{n}\right| \leq \frac{1}{\sqrt{2}}, \quad\left|\delta_{n}\right| \leq \frac{1}{\sqrt{2}}$ and $\psi_{n}=$ $\varphi_{n}-\epsilon_{n} P_{2} \varphi_{n}-\delta_{n} P_{3} \varphi_{n}$. Assume $F \in H$ and $\left(F, \psi_{n}\right)=0,(n=1,2,3, \ldots)$. Then

$$
\left(F, \varphi_{n}\right)=\epsilon_{n}\left(F, P_{2} \varphi_{n}\right)+\delta_{n}\left(F, P_{3} \varphi_{n}\right)=\epsilon_{n}\left(P_{2} F, \varphi_{n}\right)+\delta_{n}\left(P_{3} F, \varphi_{n}\right),
$$

Glasgow Math. J. 20 (1979) 49-53. 
Therefore, using $(a+b)^{2} \leq 2\left(a^{2}+b^{2}\right)$, we have

$$
\begin{aligned}
\left(F, \varphi_{n}\right)^{2} & =\left(\epsilon_{n}\left(P_{2} F, \varphi_{n}\right)+\delta_{n}\left(P_{3} F, \varphi_{n}\right)\right)^{2} \\
& \leq 2\left(\epsilon_{n}^{2}\left(P_{2} F, \varphi_{n}\right)^{2}+\delta_{n}^{2}\left(P_{3} F, \varphi_{n}\right)^{2}\right) \\
& \leq\left(P_{2} F, \varphi_{n}\right)^{2}+\left(P_{3} F, \varphi_{n}\right)^{2}
\end{aligned}
$$

Thus

or

$$
\begin{aligned}
\|F\|^{2} & =\sum_{n=1}^{\infty}\left(F, \varphi_{n}\right)^{2} \\
& \leq \sum_{n=1}^{\infty}\left(P_{2} F, \varphi_{n}\right)^{2}+\sum_{n=1}^{\infty}\left(P_{3} F, \varphi_{n}\right)^{2} \\
& =\left\|P_{2} F\right\|^{2}+\left\|P_{3} F\right\|^{2}
\end{aligned}
$$

$$
\left\|P_{1} F\right\|^{2}+\left\|P_{2} F\right\|^{2}+\left\|P_{3} F\right\|^{2} \leq\left\|P_{2} F\right\|^{2}+\left\|P_{3} F\right\|^{2}
$$

Thus $P_{1} F=0$ and all the inequalities must be equalities. Since $(a+b)^{2}=2\left(a^{2}+b^{2}\right)$ if and only if $a=b$, (1) gives us

$$
\epsilon_{n}\left(P_{2} F, \varphi_{n}\right)=\delta_{n}\left(P_{3} F, \varphi_{n}\right) \text { for all } n
$$

(2) gives us

$$
2 \epsilon_{n}^{2}\left(P_{2} F, \varphi_{n}\right)^{2}=\left(P_{2} F, \varphi_{n}\right)^{2}
$$

and

$$
2 \delta_{n}^{2}\left(P_{3} F, \varphi_{n}\right)^{2}=\left(P_{3} F, \varphi_{n}\right)^{2} \text { for all } n \text {. }
$$

Finally $\left(F, \varphi_{n}\right)=\epsilon_{n}\left(P_{2} F, \varphi_{n}\right)+\delta_{n}\left(P_{3} F, \varphi_{n}\right)$ and $P_{1} F=0$ give us

$$
\left(1-\epsilon_{n}\right)\left(P_{2} F, \varphi_{n}\right)=\left(\delta_{n}-1\right)\left(P_{3} F, \varphi_{n}\right) \text { for all } n,
$$

and we note that neither $\left(1-\epsilon_{n}\right)$ nor $\left(\delta_{n}-1\right)$ can be zero. Thus, for each $n$, either $\left(P_{2} F, \varphi_{n}\right)$ and $\left(P_{3} F, \varphi_{n}\right)$ are both zero or neither is zero.

If, for some $n$, neither $\left(P_{2} F, \varphi_{n}\right)$ nor $\left(P_{3} F, \varphi_{n}\right)$ are zero (4) and (5) give us $\left|\epsilon_{n}\right|=\left|\delta_{n}\right|=$ $1 / \sqrt{ } 2$, (3) gives us $\left|\left(P_{2} F, \varphi_{n}\right)\right|=\left|\left(P_{3} F, \varphi_{n}\right)\right|$ and (6) gives us $\left|1-\epsilon_{n}\right|=\left|\delta_{n}-1\right|$. Thus $\epsilon_{n}$ and $\delta_{n}$ must have the same sign. Then (3) tells us $\left(P_{2} F, \varphi_{n}\right)$ and $\left(P_{3} F, \varphi_{n}\right)$ have the same sign while (6) tells us they must have opposite signs. We thus conclude that, for each $n$, $\left(P_{2} F, \varphi_{n}\right)$ and $\left(P_{3} F, \varphi_{n}\right)$ are zero and the theorem is proven.

COROLlaRY. Let $\left\{a_{n}\right\}_{n=1}^{\infty}$ and $\left\{b_{n}\right\}_{n=1}^{\infty}$ be sequences such that $0<m_{1} \leq a_{n} \leq M_{1}$ and $0<m_{2} \leq b_{n} \leq M_{2}$ where $M_{i} \leq(\sqrt{ } 2+1) /(\sqrt{ } 2-1) m_{i}(i=1,2)$. If $\psi_{n}=P_{1} \varphi_{n}+a_{n} P_{2} \varphi_{n}+b_{n} P_{3} \varphi_{n}$, then $\left\{\psi_{n}\right\}_{n=1}^{\infty}$ is complete in $H$.

Proof. Assume $F$ is orthogonal to $\left\{\psi_{n}\right\}_{n=1}^{\infty}$. Then

$$
P_{1} F+\frac{M_{1} \sqrt{ } 2}{1+\sqrt{2}} P_{2} F+\frac{M_{2} \sqrt{ } 2}{1+\sqrt{ } 2} P_{3} F
$$


is orthogonal to

$$
P_{1} \varphi_{n}+\frac{1+\sqrt{ } 2}{M_{1} \sqrt{2}} a_{n} P_{2} \varphi_{n}+\frac{1+\sqrt{ } 2}{M_{2} \sqrt{2}} b_{n} P_{3} \varphi_{n}
$$

However, by Theorem 1,

$$
P_{1} \varphi_{n}+\frac{1+\sqrt{ } 2}{M_{1} \sqrt{2}} a_{n} P_{2} \varphi_{n}+\frac{1+\sqrt{ } 2}{M_{2} \sqrt{2}} b_{n} P_{3} \varphi_{n}
$$

is complete in $H$ and hence

$$
P_{1} F+\frac{M_{1} \sqrt{ } 2}{1+\sqrt{ } 2} P_{2} F+\frac{M_{2} \sqrt{ } 2}{1+\sqrt{2}} P_{3} F
$$

is zero which says that $F$ is zero.

We now turn to a generalization of Theorem B. As before an unexpected factor of $(\sqrt{ } 2+1) /(\sqrt{ } 2-1)$ arises.

THEOREM 2. Let $\psi_{n}=P_{1} \varphi_{n}+a_{n} P_{2} \varphi_{n}+b_{n} P_{3} \varphi_{n}$ where $1-1 / \sqrt{ } 2<m_{1} \leq a_{n} \leq M_{1}<$ $1+1 / \sqrt{ } 2$ and $1-1 / \sqrt{ } 2<m_{2} \leq b_{n} \leq M_{2}<1+1 / \sqrt{ } 2$. Then $\left\{\psi_{n}\right\}_{n=1}^{\infty}$ is an $\ell^{2}$ basis in $H$.

Proof. Let $a_{n}=1+\epsilon_{n}$ and $b_{n}=1+\delta_{n}$. Then $\left|\epsilon_{n}\right| \leq \epsilon<1 / \sqrt{ } 2$ and $\left|\delta_{n}\right| \leq \delta<1 / \sqrt{ } 2$ where $\epsilon=\max \left(\left|m_{1}-1\right|,\left|M_{1}-1\right|\right)$ and $\delta=\max \left(\left|m_{2}-1\right|,\left|M_{2}-1\right|\right)$. Also $\psi_{n}=\varphi_{n}+\epsilon_{n} P_{2} \varphi_{n}+\delta_{n} P_{3} \varphi_{n}$. We consider the linear map $T$ taking $H$ into $H$ defined by $T \varphi_{n}=\psi_{n}(n=1,2, \ldots)$. For any $F \in H$ we have $F=\sum_{n=1}^{\infty} F_{n} \varphi_{n}$. Therefore

$$
\begin{aligned}
\|T F\| & =\left\|T \sum_{n=1}^{\infty} F_{n} \varphi_{n}\right\| \\
& =\left\|\sum_{n=1}^{\infty} F_{n} \varphi_{n}+\sum_{n=1}^{\infty} \epsilon_{n} F_{n} P_{2} \varphi_{n}+\sum_{n=1}^{\infty} \delta_{n} F_{n} P_{3} \varphi_{n}\right\| \\
& \leq\left\|\sum_{n=1}^{\infty} F_{n} \varphi_{n}\right\|+\left\|P_{2} \sum_{n=1}^{\infty} \epsilon_{n} F_{n} \varphi_{n}\right\|+\left\|P_{3} \sum_{n=1}^{\infty} \delta_{n} F_{n} \varphi_{n}\right\| \\
& \leq\|F\|+\left\|\sum_{n=1}^{\infty} \epsilon_{n} F_{n} \varphi_{n}\right\|+\left\|\sum_{n=1}^{\infty} \delta_{n} F_{n} \varphi_{n}\right\| \\
& =\|F\|+\sqrt{ }\left(\sum_{n=1}^{\infty} \epsilon_{n}^{2} F_{n}^{2}\right)+\sqrt{\left(\sum_{n=1}^{\infty} \delta_{n}^{2} F_{n}^{2}\right)} \\
& \leq\|F\|+\epsilon\|F\|+\delta\|F\| \\
& \leq(1+\sqrt{ } 2)\|F\|
\end{aligned}
$$

Thus $\|T\| \leq 1+\sqrt{ } 2$. 
We also have, for each $F \in H$,

$$
\begin{aligned}
\|T F\| & =\left\|\sum_{n=1}^{\infty} F_{n} \varphi_{n}+\sum_{n=1}^{\infty} \epsilon_{n} F_{n} P_{2} \varphi_{n}+\sum_{n=1}^{\infty} \delta_{n} F_{n} P_{3} \varphi_{n}\right\| \\
& \geq\left\|\sum_{n=1}^{\infty} F_{n} \varphi_{n}\right\|-\left\|P_{2} \sum_{n=1}^{\infty} \epsilon_{n} F_{n} \varphi_{n}+P_{3} \sum_{n=1}^{\infty} \delta_{n} F_{n} \varphi_{n}\right\| \\
& \geq\|F\|-\sqrt{ }\left(\epsilon^{2}+\delta^{2}\right)\left\|\sum_{n=1}^{\infty} F_{n} \varphi_{n}\right\| \\
& =\left(1 \sqrt{ }\left(\epsilon^{2}+\delta^{2}\right)\right)\|F\|
\end{aligned}
$$

(and we note that $1-\sqrt{ }\left(\epsilon^{2}+\delta^{2}\right)>0$ ). By standard theory of linear operators (see [5]) $T^{-1}$ exists and is bounded on $T(H)$. However, since, by Theorem $1, T(H)$ is dense in $H$, we can extend $T^{-1}$ to be a bounded operator on $H$. Consequently, given $F \in H$ we can write $F=T g$ for a unique $g \in H$. Since $g$ is uniquely of the form $\sum_{n=1}^{\infty} g_{n} \varphi_{n}$ (with $\left\{g_{n}\right\} \in \ell^{2}$ ) we have $F=T g=T\left(\sum_{n=1}^{\infty} g_{n} \varphi_{n}\right)=\sum_{n=1}^{\infty} g_{n} \psi_{n}$ and the theorem is proved.

Corollary. Let $\psi_{n}=P_{1} \varphi_{n}+a_{n} P_{2} \varphi_{n}+b_{n} P_{3} \varphi_{n}$ where $0<m_{1} \leq a_{n} \leq M_{1}, 0<m_{2} \leq b_{n} \leq$ $M_{2}$ and $M_{1}<(\sqrt{2}+1) /(\sqrt{2}-1) m_{i}(i=1,2)$. Then $\left\{\psi_{n}\right\}_{n=1}^{\infty}$ is an $\ell^{2}$ basis in $H$.

Proof. For $i=1,2$, choose $C_{i}$ such that

$$
\frac{\sqrt{2}-1}{\sqrt{2 m_{i}}}<C_{i}<\frac{\sqrt{2}+1}{\sqrt{2 M_{i}}}
$$

Then by Theorem 2, $\left\{P_{1} \varphi_{n}+C_{1} a_{n} P_{2} \varphi_{n}+C_{2} b_{n} P_{3} \varphi_{n}\right\}$ is an $\ell^{2}$ basis in $H$. Thus given any $F \in H$ there is a unique sequence $\left\{F_{n}\right\}_{n=1}^{\infty} \in \ell^{2}$ such that

$$
P_{1} F+C_{1} P_{2} F+C_{2} P_{3} F=\sum_{n=1}^{\infty} F_{n}\left(P_{1} \varphi_{n}+C_{1} a_{n} P_{2} \varphi_{n}+C_{2} b_{n} P_{3} \varphi_{n}\right)
$$

or equivalently, $F=\sum_{n=1}^{\infty} F_{n} \psi_{n}$ which was to be proved.

COUNTEREXAMPLE. Upon comparing Theorems 1 and 2 (and their corollaries) with Theorems $\mathrm{A}$ and $\mathrm{B}$, we notice that Theorems $\mathrm{A}$ and $\mathrm{B}$ are not generalized as completely as would be expected. For example, in Theorem B we have $0<m \leq a_{n} \leq M$ which, in the case of triple series, we would expect to become

$$
0<m_{1} \leq a_{n} \leq M_{1} \text { and } 0<m_{2} \leq b_{n} \leq M_{2}
$$

Instead, as in the corollary to Theorem 2, we have an additional factor of $M_{i}<$ $(\sqrt{ } 2+1) /(\sqrt{ } 2-1) m_{i}(i=1,2)$. As a means of proving that some additional condition is needed, we have the following counterexample. For $n=1,2, \ldots$ let $\varphi_{n}(x)=\sin n x$. Let 
$H=L^{2}[0, \pi], \quad \mathbf{P}_{1}=L^{2}[0,5 \pi / 16], \quad \mathbf{P}_{2}=L^{2}[5 \pi / 16, \pi / 2]$, and $\mathbf{P}_{3}=L^{2}[\pi / 2, \pi]$. For $n=$ $4,5,6, \ldots$ let $a_{n}=b_{n}=1$; i.e. $\psi_{n}(x)=\varphi_{n}(x)=\sin n x$. Let $t$ be a real number, $a_{1}=b_{1}=b_{2}=$ $a_{3}=t$ and let $a_{2}=b_{3}=2-t$. Thus for all $t \in(0,1)$ we have $0<t \leq a_{n} \leq 2-t$ and $0<t \leq$ $b_{n} \leq 2-t$. Thus for all $t \in(0,1)\left\{a_{n}\right\}$ and $\left\{b_{n}\right\}$ would satisfy condition $\left(^{*}\right)$. However, we shall show that for some $t \in(0,1)$ not only is $\left\{\psi_{n}\right\}$ not an $\ell^{2}$ basis but it is not even complete in $H$.

In searching for a function $F(x) \in L^{2}[0, \pi]$ orthogonal to $\left\{\psi_{n}\right\}_{n=1}^{\infty}$ we write $F(x)=$ $\sum_{n=1}^{\infty} F_{n} \sin n x$. Then since, for $n=4,5, \ldots F$ is orthogonal to $\sin n x$ we have $F_{n}=0$ $(n=4,5, \ldots)$. Thus we merely need find non-zero $F_{1}, F_{2}$, and $F_{3}$ such that $\sum_{n=1}^{3} F_{n} \sin n x$ is orthogonal to $\left\{\psi_{n}\right\}_{n=1}^{3}$. We can find such $\left\{F_{i}\right\}_{i=1}^{3}$ if and only if the determinant $\left|\left(\psi_{i}(x), \sin j x\right)\right|$ is zero. Keeping in mind that the coefficients of $\left\{\psi_{i}\right\}_{i=1}^{3}$ depend on $t \in(0,1)$ we set $D(t)=\left|\left(\psi_{i}(x), \sin j x\right)\right|(i, j=1,2,3) . D(t)$ is a continuous function of $t$ and $8 D(0.07)=-0.071942$ while $8 D(0.08)=0.318591$. Thus for some $t \in(0.07,0.08), D(t)$ is zero and hence $\left\{\psi_{n}(x)\right\}_{n=1}^{\infty}$ is incomplete (and certainly not a basis) in $L^{2}[0, \pi]$ even though it satisfies $\left({ }^{*}\right)$. We note that for $t>(\sqrt{2}-1) / \sqrt{ } 2=0.29289$ we have all the hypotheses of the corollary to Theorem 2 satisfied and hence $\left\{\psi_{n}(x)\right\}_{n=1}^{\infty}$ would be an $\ell^{2}$ basis in $L^{2}[0, \pi]$. Thus while there is some gap between the $t$ of our counterexample and $t>(\sqrt{ } 2-1) / \sqrt{ } 2$ we have established that something more than $\left(^{*}\right)$ is necessary and that Theorem B is not generalized in the obvious manner.

The author would like to express his thanks and appreciation to Professor Daniel Prener for his assistance in setting up the computer program that yielded the counterexample.

\section{REFERENCES}

1. R. Feinerman and R. Kelman, The convergence of least squares approximations for dual orthogonal series, Glasgow Math. J. 15 (1974), 82-84 and Corrigenda, ibid 184.

389-502. R. Feinerman and R. Kelman, Dual orthogonal series with oscillatory modifiers, SIAM J. Math. Anal. 9 (1978), 591-594.

4. R. Feinerman, Dual orthogonal series with modifier tending to zero, SIAM J. Math. Anal. 9 (1978), 667-670.

5. G. Bachman and L. Narici, Functional analysis, (Academic Press, 1966).

Department of Mathematics

Herbert H. Lehman College (CUNY)

Bronx, New York 10468

Present address:

Department of Pure Mathematics

Weizmann InstituTE

REHOVOT, ISRAEL 